\title{
Population Genetic Structure of the German Cockroach (Blattodea: Blattellidae) in Apartment Buildings
}

\author{
JONATHAN R. CRISSMAN, ${ }^{1,2}$ WARREN BOOTH,${ }^{1,2}$ RICHARD G. SANTANGELO,${ }^{1}$ \\ DMITRY V. MUKHA,${ }^{3}$ EDWARD L. VARGO,${ }^{1}$ AND COBY SCHAL ${ }^{1,4}$
}

\begin{abstract}
J. Med. Entomol. 47(4): 553-564 (2010); DOI: 10.1603/ME09036
ABSTRACT The German cockroach, Blattella germanica (L.) (Blattodea: Blattellidae), is a major residential pest with the potential to vector various pathogens and produce and disseminate household allergens. Understanding population genetic structure and differentiation of this important pest is critical to efforts to eradicate infestations, yet little is known in this regard. Using highly polymorphic microsatellite markers, we investigated patterns of genetic diversity and differentiation within and among 18 apartments from six apartment complexes located in Raleigh, NC. No departure from panmixia was found between rooms within apartments, indicating that active dispersal resulting in gene flow may occur among rooms within apartment units. Alternatively, aggregations within apartments may exist in relative isolation under a metapopulation framework, derived from a recent, common source. Thus, in the event of population control practices leading to incomplete cockroach eradication within an apartment, recolonization of shelters and rooms is likely to occur from a genetically similar aggregation. A pattern of isolation-by-distance across the six apartment complexes indicated that dispersal was more common within complexes than among them, and $F$ statistics suggested greater genetic similarity between apartments in a single building than between separate buildings of an apartment complex. Similarly, neighbor-joining tree and Bayesian clustering analyses were able to cluster only those apartments that were within a single building, indicating higher dispersal with associated gene flow within buildings than between them. The lack of any broader connectivity, as indicated by significant $F_{\mathrm{ST}}$ and $G$-tests suggests that human-mediated dispersal of $B$. germanica between buildings of an apartment complex or between complexes occurs infrequently enough to have negligible effects on gene flow.
\end{abstract}

KEY WORDS Blattella germanica, genetic diversity, human-mediated dispersal, metapopulation, microsatellite

The German cockroach, Blattella germanica (L.) (Blattodea: Blattellidae), is widely considered the most prominent of the household pest cockroaches, and is a ubiquitous pest of human-built structures in cosmopolitan regions on every continent (Appel 1995). It is an economically and medically important pest, and is a capable vector of various pathogens including bacteria (Fotedar et al. 1991, Cloarec et al. 1992, Zurek and Schal 2004, Pai et al. 2005, Elgderi et al. 2006), fungi (Fotedar and Banerjee 1992), and protozoa (Pai et al. 2003, Graczyk et al. 2005). Perhaps most importantly, this species is a major source of indoor allergens, particularly in urban environments and low socioeconomic households (Gore and Schal 2007). Exposure to high levels of cockroach allergens in the home leads to greater allergenic sensitization

${ }^{1}$ Department of Entomology and W. M. Center for Behavioral Biology, Box 7613, North Carolina State University, Raleigh, NC 27695-7613.

${ }^{2}$ These authors contributed equally to this article.

${ }^{3}$ Vavilov Institute of General Genetics, Russian Academy of Sciences, Gubkin 3, Moscow 119991, Russia.

${ }^{4}$ Corresponding author, e-mail: coby schal@ncsu.edu.
(Eggleston et al. 1998) and has been linked to significant increases in asthma morbidity among inner city children (Rosenstreich et al. 1997). Arbes et al. (2004) showed that a significant reduction of cockroach allergen levels could be achieved by cockroach control alone. A clearer understanding of how cockroach populations are connected within apartments, between buildings and beyond, may therefore represent a significant step forward in the development of control strategies and in understanding of the success or failure of such strategies for this commensal pest.

The unique, obligatory commensal ecology of $B$. germanica suggests that variation is likely to exist in its dispersal within and between structures. It is particularly well adapted to the indoor environment and is not known to exist in either natural or outdoor environments anywhere in its distribution (Roth 1985), suggesting that dispersal should be more likely to occur within a building than between-buildings separated by open habitat. Therefore, active dispersal is likely within buildings, but between building movement probably requires human mediation. Most empirical studies have focused on the ability of B. ger- 


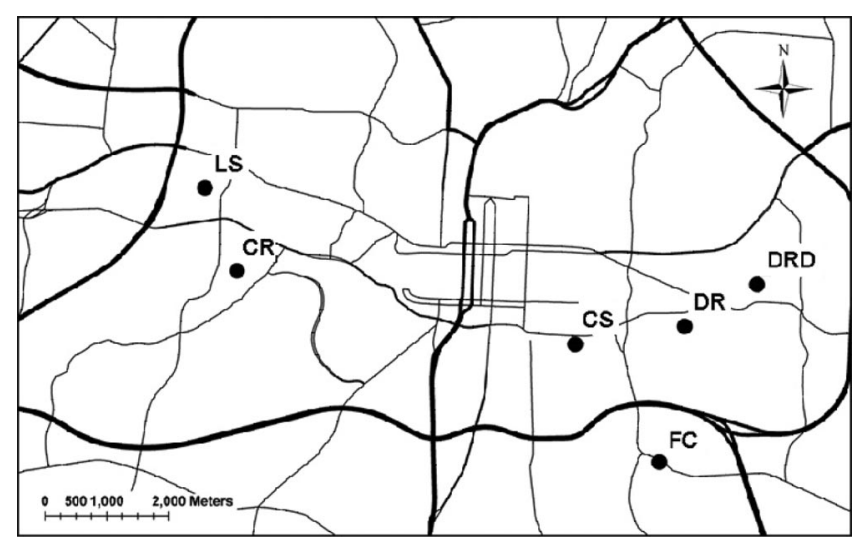

Fig. 1. Geographic location of apartment complexes from which B. germanica were sampled within Raleigh, NC. Lines represent major roads.

manica to disperse within a single building, with a few studies focused on apartment buildings. Using markrecapture methods Owens and Bennett (1982) found that over a 3 -wk period, up to $32 \%$ of recaptured $B$. germanica had moved from another room within the same apartment. A comparable number were found to have moved between adjacent apartments. These results were somewhat contradicted by two subsequent studies, however. Runstrom and Bennett $(1984,1990)$ found much lower immigration from adjacent apartments, up to 9 and $13 \%$ of recaptures, respectively. As with most mark-recapture studies, however, these suffered from low recapture rates which declined rapidly with distance from the point of release. Although these studies found that most recaptured cockroaches exhibited site fidelity to their original capture area, they illustrate the potential for gene flow both within and between apartments.

Data describing the genetic structure of $B$. germanica populations are limited. Despite significant geographic separation, no genetic differentiation was detected between B. germanica populations sampled from two cities in France when analyzed at eight allozyme loci (Cloarec et al. 1999). Furthermore, phylogenetic clustering analysis did not detect city-specific sample association. A follow-up study (Jobet et al. 2000) using 10 randomly amplified polymorphic DNA markers found weak genetic differentiation among samples from the same two cities sampled by Cloarec et al. (1999). Again, samples could not be separated according to city. The results of both studies are contrary to the hypothesis that greater gene flow would occur more frequently between geographically proximate populations. In both cases, low diversity of the genetic markers precluded any fine-scale analysis of genetic structure within a city.

The goal of this study was to investigate population genetic differentiation of $B$. germanica at hierarchical spatial scales ranging from the single apartment level, to samples collected across a city, by using highly polymorphic microsatellite markers developed for this species (Booth et al. 2007). We tested the hypotheses that differentiation will be limited both within apart- ments and within buildings due to the narrow active dispersal capability of B. germanica. Significant genetic differentiation, however, should exist between buildings due to the likelihood that human-mediated dispersal is sufficiently infrequent to allow genetic divergence to arise.

\section{Materials and Methods}

Sample Collection. Cockroaches were collected in a hierarchical manner to address the issue of gene flow and differentiation at several spatial scales. Samples were collected from multiple rooms within three apartments $(n=5$ per apartment), from three apartments per complex ( $n=3$ complexes), and from six complexes within the city of Raleigh, NC (Fig. 1). Infested homes were identified by a survey of residents, and cockroaches were captured using unbaited sticky traps deployed for 24 or $48 \mathrm{~h}$ between October 2006 and January 2008. Samples were immediately preserved in $95 \%$ ethanol and kept at $-20^{\circ} \mathrm{C}$ pending DNA extraction. Within each apartment, cockroach infestations were considered to be moderate to heavy, with numbers estimated to be in the thousands to the tens of thousands. Sample location names have been modified to provide anonymity to the communities within which samples were collected.

Within apartments. To investigate gene flow and differentiation between rooms of an apartment, multiple traps were placed overnight in the kitchen $(n=$ 2 traps), living room ( $n=1$ trap), and bedroom ( $n=$ 1 trap). From these traps, four samples of 30 adult $B$. germanica were selected for genetic analysis. A fifth sample of live individuals was collected during the day near one of the kitchen traps using a modified vacuum. This sample allowed us to verify that cockroaches trapped on a particular trap were genetically representative of the local aggregation. Multiple-room sampling was replicated in three apartment complexes (FC, DR, and DRD), and these apartments were labeled apartment A for each apartment complex (e.g., FC-A). 
Within buildings. For inference on population structure between apartments in a single building, three apartments were sampled from each of three low-rise apartment complexes (LS, CS, and CR), as equivalent samples were not available from complexes FC, DR, and DRD. Samples were taken from sticky traps placed in the kitchen of each apartment. Apartments were chosen such that two apartments, labeled $\mathrm{A}$ and $\mathrm{B}$, were adjoining and shared a wall, and the third, labeled C, was located elsewhere in the building.

Between buildings. To investigate genetic differentiation between buildings in an apartment complex, two additional apartments from separate buildings were sampled from apartment complexes FC, DR, and DRD. Samples were taken from sticky traps placed in the kitchen of each apartment. These apartments were labeled B and C.

Microsatellite Genotyping. DNA was extracted from the legs and thorax of each individual using the Gentra Puregene DNA extraction kit (Gentra Systems Inc., Minneapolis, MN). Samples were initially screened at nine microsatellite loci (Bg-1D5, Bg-A7, Bg-B12, Bg-CO4, Bg-D05, Bg-D9, Bg-F7, Bg-G7, Bgwb-2 A) according to the polymerase chain reaction (PCR) procedures outlined by Booth et al. (2007). Amplified products were labeled with M13 F-29 IRDye infrared tags (Li-Cor Biosciences, Lincoln, NE). Samples were loaded on $25 \mathrm{~cm} 6 \% 1 \times$ TBE polyacrylamide gels and run on a 4300 automated DNA sequencer (Li-Cor Biosciences). Fifty- to 350-bp IRDye size standards (Li-Cor Biosciences) were loaded every 15 samples for accurate size determination. At least one control sample (i.e., a sample of known genotype) was included in each run to ensure accuracy and consistency of scoring among different gels. Allele sizes were scored using Gene Profiler software, version 4.05 (Scanalytics, Inc., BD Biosciences Bioimaging, Rockville, MD).

Genetic Data Analysis. Samples were analyzed hierarchically to take into consideration the aims outlined above. Summary population statistics (mean number of alleles $\left[N_{\mathrm{A}}\right]$, expected $\left[H_{\mathrm{E}}\right]$, and observed $\left[H_{\mathrm{O}}\right]$ heterozygosities) were calculated using the Genetic Data Analysis software (Lewis and Zaykin 2001). Departures from Hardy-Weinberg equilibrium (HWE) and genotypic linkage equilibrium were tested for each sample across all loci in GENEPOP, version 4.0 (Raymond and Rousset 1995, Rousset 2008). Bonferroni correction for multiple tests was applied to each of these tests. MICRO-CHECKER, version 2.2.3 software (Van Oosterhout et al. 2004) was used to assess the likelihood that null alleles, scoring error, or large allele drop out were evident at any locus screened.

Evidence of departures from panmixia between samples and the associated levels of genetic differentiation was assessed among samples from discrete sites by using several independent approaches. Pairwise genotypic differentiation was tested using the loglikelihood based G-test (Goudet et al. 1996), implemented in GENEPOP. The Markov Chain parameters were set to 2,000 dememorizations, 200 batches, and
2,000 iterations per batch. Departures from panmixia were used to group samples into populations following the method proposed by Waples and Gaggiotti (2006), whereby samples were considered to be part of the same population when they could be connected to any of the other samples by a nonsignificant $G$-test $(P>$ $0.05)$. At each spatial scale, differentiation was assessed by global and pairwise $F_{\mathrm{ST}}$ values (Weir and Cockerham 1984) computed using FSTAT, version 2.9.3.2 (Goudet 1995). Confidence intervals for global $F_{\text {ST }}$ values were provided by bootstrapping over loci.

To assess the patterns of genetic relationships within, between and among the 18 sampled sites, factorial correspondence analysis (FCA) based on allele frequency data were used. (after estimation of genetic differentiation using $F_{\mathrm{ST}}$, no significant differentiation was detected between the five within-apartment samples of each of the three apartments sampled. As a result, a single sample was employed for further analysis; see Results). Three-dimensional FCA plots were generated using the program GENETIX, version 4.03 (Belkhir et al. 1996-2004). We also constructed an unrooted neighbor-joining ( $\mathrm{NJ}$ ) tree based on the chord distance $\left(D_{\mathrm{C}}\right)$ of Cavalli-Sforza and Edwards (1967) by using the program MICROSAT (Minch et al. 1995). This distance was chosen as it has been shown to perform best when reconstructing tree topologies based on microsatellite data (Takezaki and Nei 1996). The 8,911 pseudoreplicate distance matrices generated were subjected to cluster analysis using NEIGHBOR in Phylip, version 3.573 (Felsenstein $1985 \mathrm{~b}$ ), implementing sample randomization to construct dendrograms. The consensus tree was obtained using CONSENSE within Phylip with the reliability of tree nodes evaluated by bootstrap analysis (Felsenstein 1985a).

A test of isolation-by-distance was used to determine whether genetic differentiation and geographic distance were correlated across the 18 apartment samples. The test was performed by regression analysis of the genetic and geographic distances for each pair of populations using the Mantel test (Mantel 1967) implemented in MANTEL, version 2 (Liedloff 1999), with a total of 10,000 permutations. Geographic distances, calculated as the shortest straight line distance between each pair of apartments, were $\log$ transformed and genetic distances transformed to $F_{\mathrm{ST}} / 1-F_{\mathrm{ST}}$.

Underlying genetic structure across Raleigh was investigated using the Bayesian clustering program STRUCTURE, version 2.2.3 (Pritchard et al. 2000). STRUCTURE estimates allele frequencies for each of a user-defined number of clusters $(K)$ and simultaneously assigns fractions of each individual's genome to one or more clusters without considering a priori information about their sampling origin. The process is repeated for various values of $K$ to determine the value with the highest likelihood of explaining the genetic variation across all individuals. Runs were based on 500,000 iterations after a 100,000 burn-in period, with values of $K$ ranging from 1 through 24 . Although 18 apartments were sampled, we tested 
Table 1. Genetic diversity statistics across eight microsatellite loci for within-apartment B. germanica samples

\begin{tabular}{|c|c|c|c|c|c|c|}
\hline Sample $^{a}$ & $N$ & $N_{\mathrm{A}}$ & $H_{\mathrm{E}}$ & $H_{\mathrm{O}}$ & $\mathrm{HWE}^{b}$ & $F_{\mathrm{ST}}(95 \% \mathrm{CI})$ \\
\hline FC-A K1 & 29 & 7.38 & 0.74 & 0.66 & ns & \\
\hline FC-A K2 & 30 & 8.13 & 0.74 & 0.71 & ns & \\
\hline FC-A vKl & 30 & 8.38 & 0.76 & 0.72 & ns & \\
\hline FC-A LR & 30 & 7.75 & 0.72 & 0.73 & ns & \\
\hline FC-A BR & 30 & 7.75 & 0.74 & 0.71 & ns & \\
\hline Across all rooms & & & & & & $0.002(-0.003,0.009)$ \\
\hline DR-A K1 & 30 & 7.75 & 0.70 & 0.63 & ns & \\
\hline DR-A K2 & 30 & 7.88 & 0.71 & 0.65 & ns & \\
\hline DR-A vK2 & 30 & 7.88 & 0.72 & 0.74 & ns & \\
\hline DR-A LR & 30 & 7.00 & 0.70 & 0.71 & ns & \\
\hline DR-A BR & 30 & 7.75 & 0.67 & 0.63 & $*$ & \\
\hline Across all rooms & & & & & & $0.002(-0.002,0.006)$ \\
\hline DRD-A K1 & 30 & 6.75 & 0.71 & 0.71 & ns & \\
\hline DRD-A K2 & 30 & 6.88 & 0.70 & 0.66 & ns & \\
\hline DRD-A vK2 & 30 & 7.25 & 0.71 & 0.72 & ns & \\
\hline DRD-A LR & 30 & 6.5 & 0.70 & 0.70 & ns & \\
\hline DRD-A BR & 21 & 6.25 & 0.71 & 0.68 & ns & \\
\hline Across all rooms & & & & & & $-0.004(-0.007,0.000)$ \\
\hline
\end{tabular}

${ }^{a} \mathrm{FC}, \mathrm{DR}$, and DRD are three different apartment complexes. A represents an apartment within each complex. $\mathrm{K}$, kitchen $(1,2=\mathrm{two}$ kitchen traps); vK, kitchen vacuum sample associated with kitchen trap 1 or 2; LR, living room; BR, bedroom.

${ }^{b}$ Significance of HWE tests (Raymond and Rousset 1995): *, $P<0.05$; ns, not significant.

higher values of $K$ to determine the likelihood that substructure exists within single apartments. Model parameters had equilibrated before the end of the burn-in phase and posterior probabilities were consistent across all 10 runs for each data and parameter set, thus informing us that our estimates of the simulation were accurate. Runs were replicated a total of 10 times to check concordance of the data.
STRUCTURE analysis was performed assuming the admixture model with allele frequencies correlated. The most appropriate $K$ value was determined using the $\Delta K$ method of Evanno et al. (2005). The 10 replicated STRUCTURE runs for the optimal $\Delta K$ were aligned using the software CLUMPP (Jakobsson and Rosenberg 2007). Replicated runs were aligned maximizing similarities between each individual's mem-

Table 2. Genetic diversity statistics across eight microsatellite loci for Raleigh apartment populations of $B$. germanica

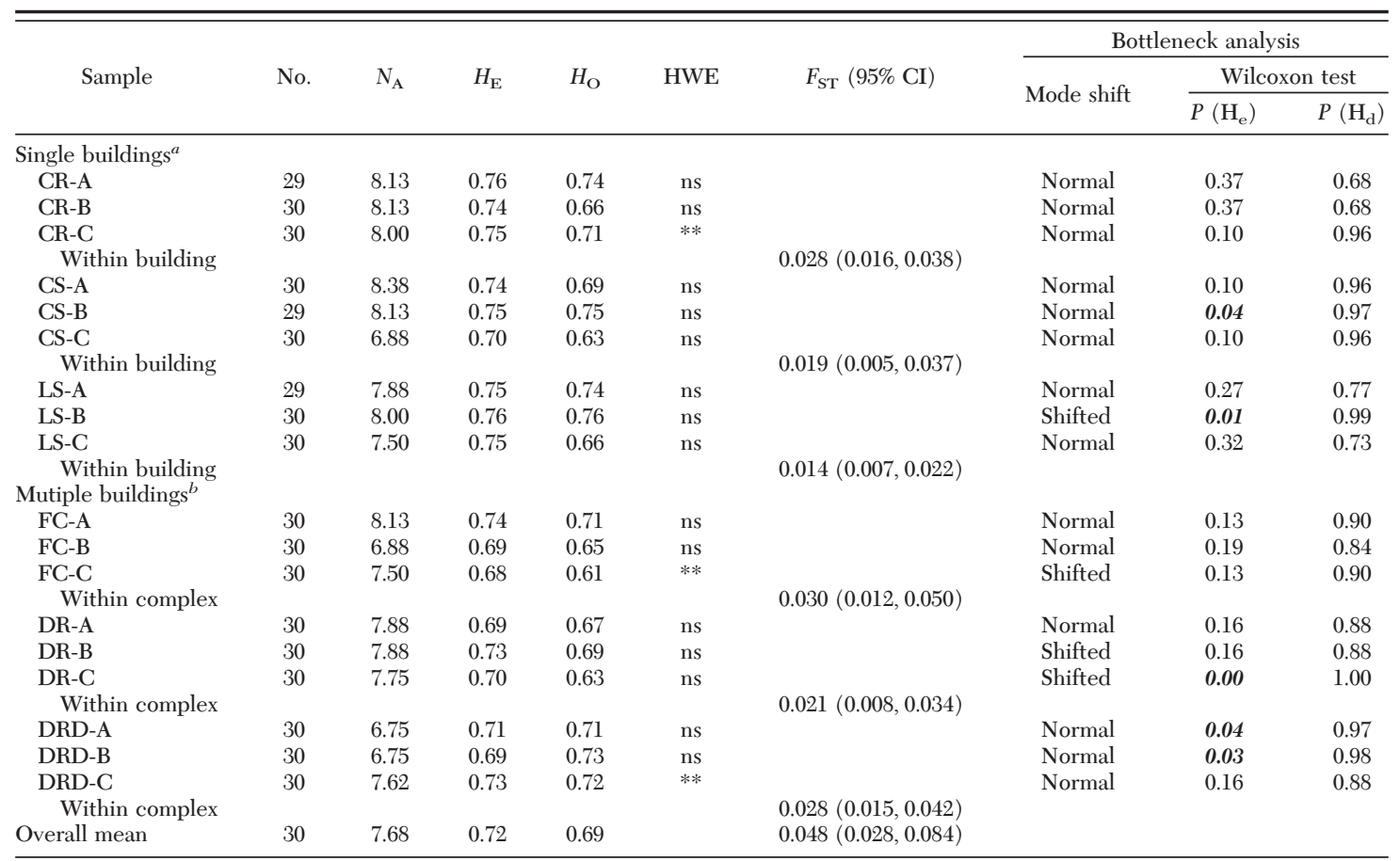

Significance of HWE tests (Raymond and Rousset 1995): **, $P<0.01$; ns, not significant.

${ }^{a}$ Single buildings: A and B represent adjacent apartments sharing a wall, and $\mathrm{C}$ represents a distant apartment within the same building.

${ }^{b}$ Multiple buildings: A, B, and C represent individual apartments within separate individual buildings within the complex. 

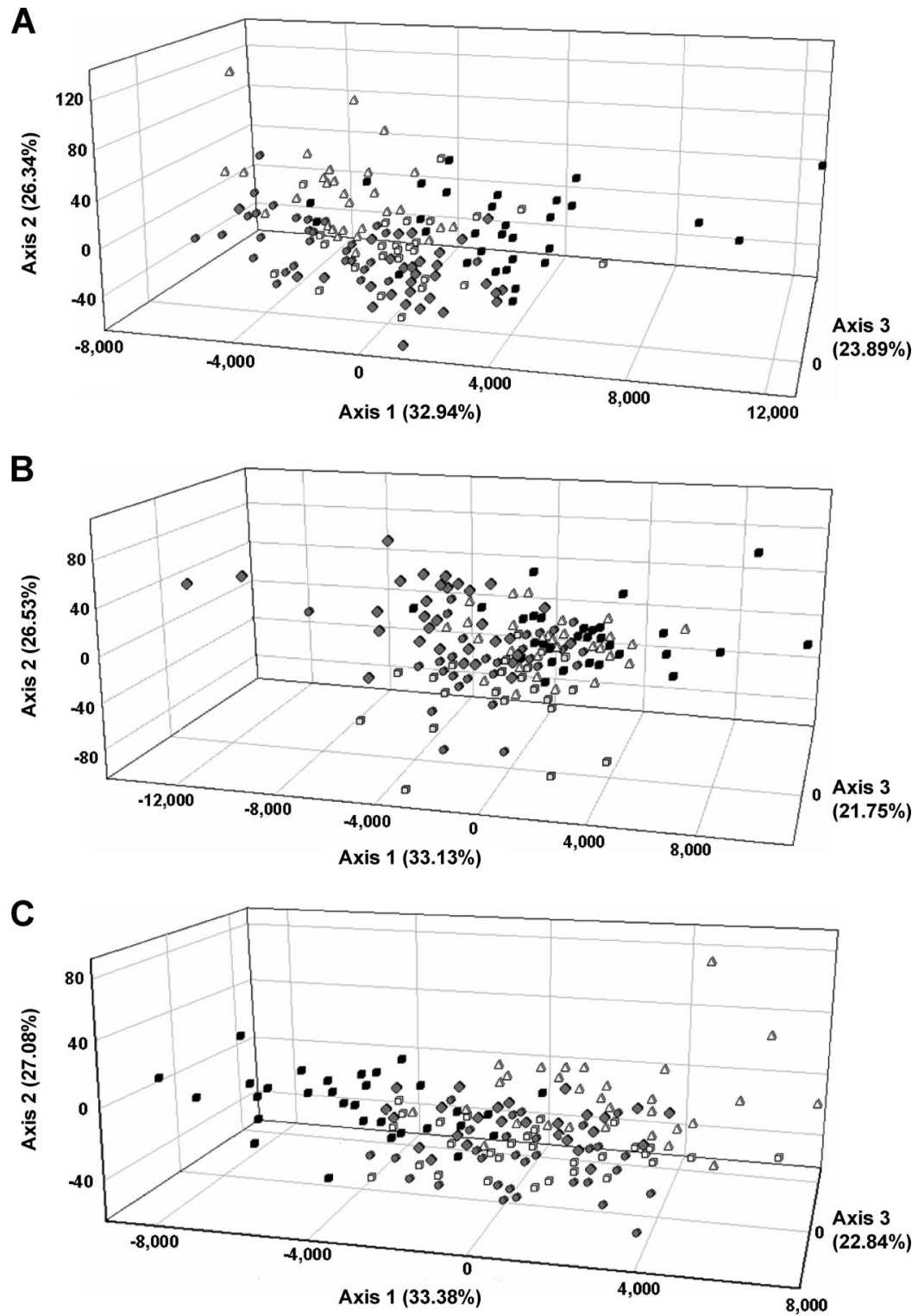

Fig. 2. Results of FCA analysis showing genetic differentiation based on microsatellite allelic frequency for individual B. germanica collections within single apartments, FC (a), DRD (b), and DR (c). Kitchen trap one (open squares), kitchen trap two (circles), kitchen vacuum sample (triangles), living room (diamonds), and bedrooms (closed squares).

bership assignments across clusters using the Greedy algorithm, with 10,000 random input orders.

Bottleneck, version 1.2.01 (Cornuet and Luikart 1996) was used to examine for evidence of recent genetic bottlenecks, through comparison of $H_{\mathrm{E}}$ and $H_{\mathrm{O}}$ under mutation-drift equilibrium. Two methods implemented in this program were used. Distortions of allele frequency distributions within samples were examined following the qualitative graphical method (i.e., mode-shift; Luikart et al. 1998). Heterozygosity excess within samples was then tested using the Wilcoxon sign rank test (Cornuet and Luikart 1996). Tests for detecting bottlenecks were assessed under the two-phase model of mutation (TPM; Di Rienzo et al. 1994), assuming a mix of 90:10\% SMM:IAM (stepwise mutation model:infinite alleles model) and $10 \%$ variance.

\section{Results}

Basic Summary Statistics. Genetic diversity was moderate to high within each of the $18 \mathrm{~B}$. germanica apartment samples collected, with observed mean heterozygosity ranging from 0.61 to 0.76 (overall mean, $0.69)$ and mean number of alleles per locus from 6.75 to 8.38 (overall mean, 7.68) (Tables 1 and 2). These 

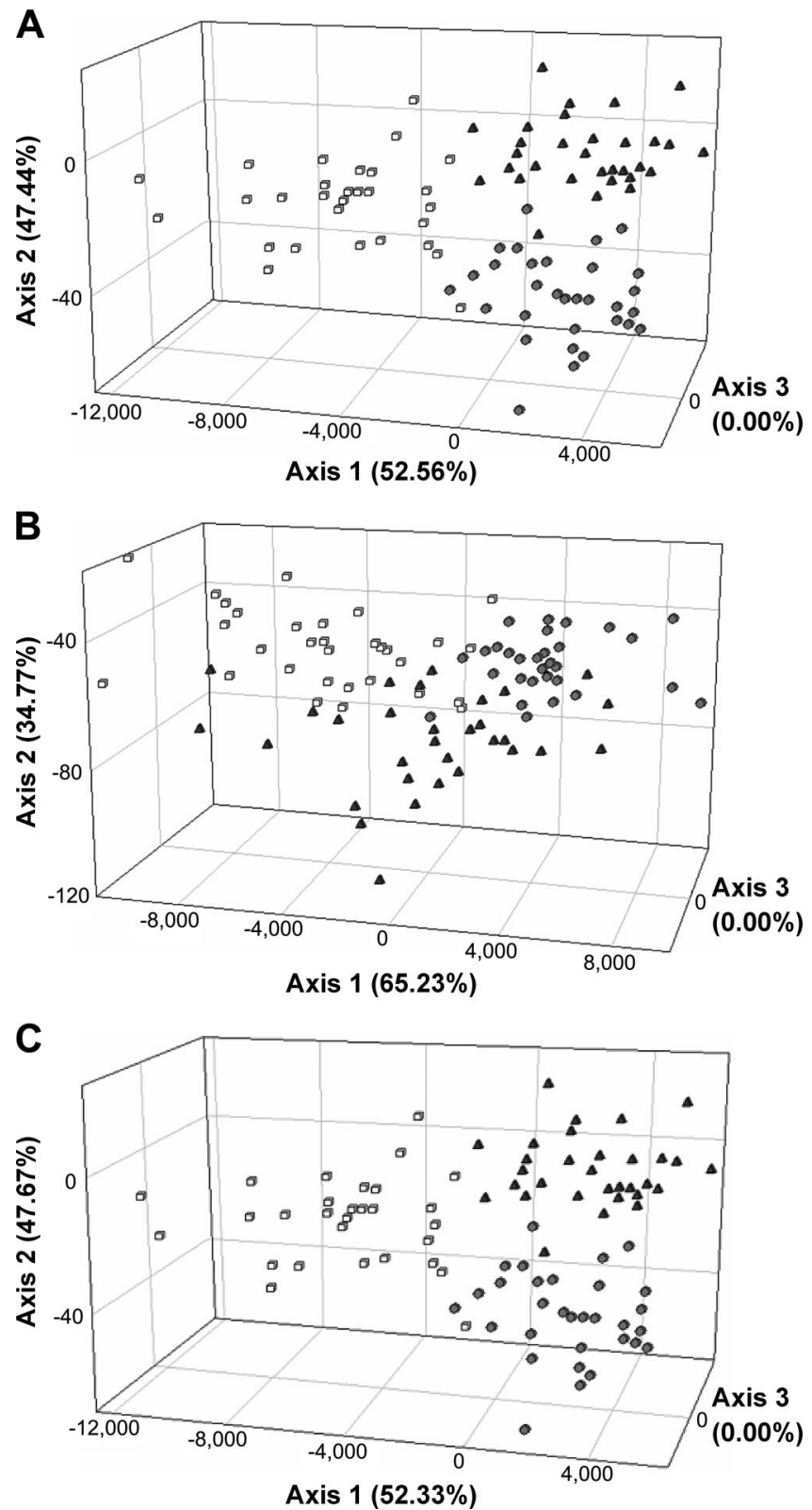

Fig. 3. Results of FCA analysis showing genetic differentiation based on microsatellite allelic frequency for individual B. germanica collections within buildings ( $a, C R ; b, C S$; and c, LS) and within apartment complexes (d, FC; e, DRD; and f, DR). Apartment A (square), apartment B (circle), and apartment C (triangle).

values are comparable, if not slightly greater, than those reported by Booth et al. (2007) for populations inhabiting hog farms within North Carolina. Within each sample, more than four alleles per locus were commonly observed. An excess of homozygotes was detected at both $\mathrm{Bg}$-C04 and Bg-D9; however, only $B g$-D9 exhibited allelic signatures characteristic of the presence of null alleles. The excess of homozygotes at Bg-C04 might have resulted from sampling error due to the large number of alleles present at this locus.
Locus $B g$-D9 was therefore excluded from further analyses.

Significant deviations from Hardy-Weinberg equilibrium were observed at a single locus, $B g$-C04, in five of the 15 multiroom apartment samples. Only one sample (DR-A BR, Table 1) deviated significantly from $\mathrm{HWE}$ when $\mathrm{Bg}$-C04 was removed from the analysis. When room samples within each apartment were combined and analyzed as a single population, no deviation from HWE was detected. As no evidence of 

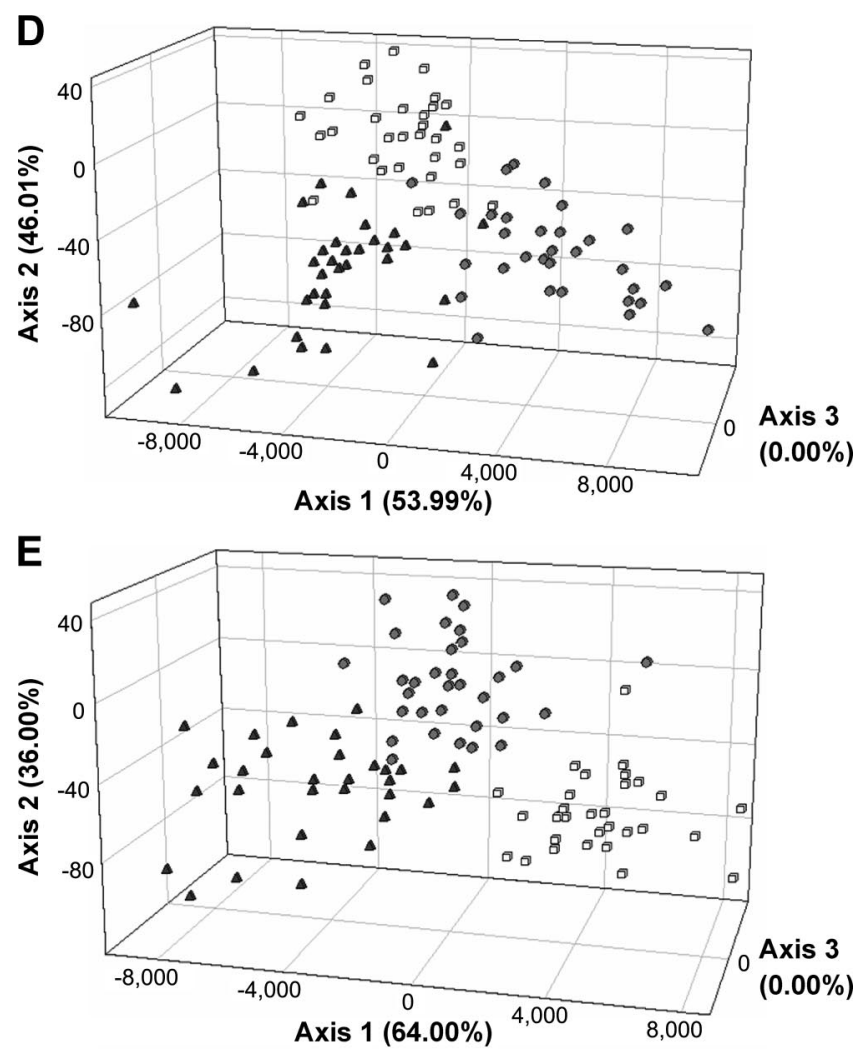

$(0.00 \%)$

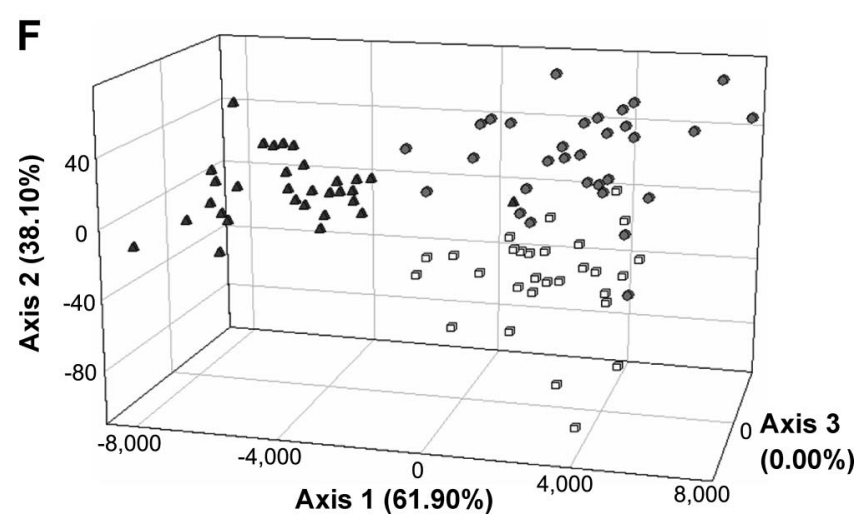

Fig. 3. (Continued).

null alleles was present at Bg-C04, it was not excluded from further analyses. Although the kitchen was often the most highly infested room in an apartment (unpublished data), allelic diversity was not significantly higher within this room compared with the bedroom and living room samples $(P=0.16$; $t$-test, two sample assuming unequal variance). Significant deviations from HWE also were detected in three additional apartments (FC-C, CR-C, and LS-C). Deviations resulted from homozygote excess at a single different locus in each case. Little evidence was found to support linkage disequilibrium between pairs of loci. Of 504 pairwise comparisons, 20 were found to be significant $(P \leq 0.05)$. These significant cases were found to be randomly distributed among populations and pairs of loci.

Population Genetic Differentiation. Within apartments. Within each apartment, nonsignificant $G$-test values connecting all five samples were found. Similarly, global $F_{\mathrm{ST}}$ values were not significantly different from zero in any of the apartments, as determined by $95 \%$ confidence intervals (Table 1 ). FCA analysis of $B$. germanica samples within all apartments revealed similar patterns, confirming the results of both the G-tests and global $F_{\mathrm{ST}}$. Each revealed considerable overlap of individuals between all sampled rooms (Fig. 2A-C). Given the lack of significant genetic differentiation between sampled rooms, confirmed through $G$-test, 


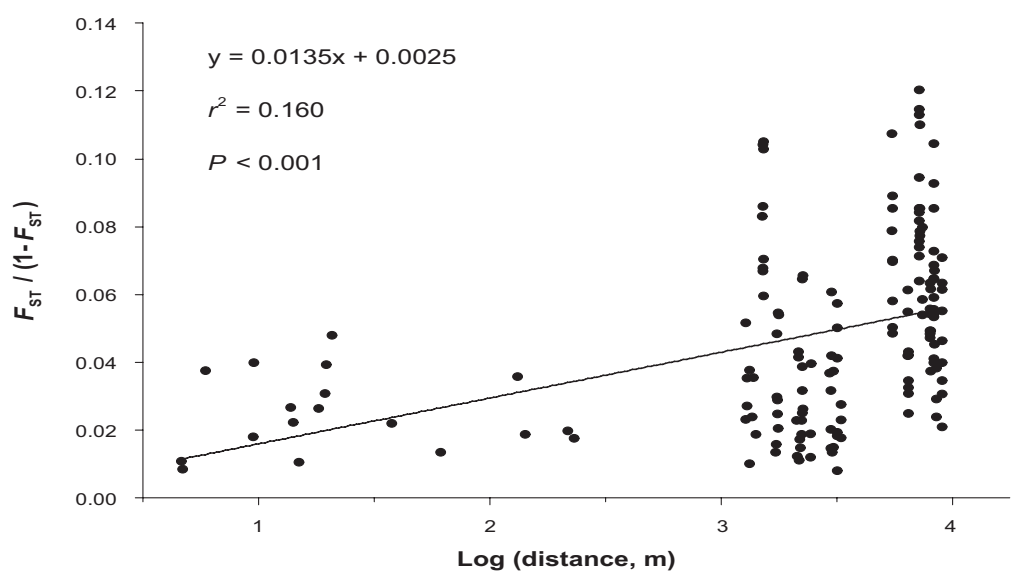

Fig. 4. Isolation by distance analysis for B. germanica apartment populations within Raleigh, NC. Pairwise comparisons between populations are plotted as genetic distance transformed as $F_{\mathrm{ST}} /\left(1-F_{\mathrm{ST}}\right)$ versus the natural log of geographic distance. The equation for the regression analysis, $r^{2}$ value, and results for the Mantel test of significance are given.

$F_{\mathrm{ST}}$ and FCA analysis, we determined that for each of the apartments FC-A, DRD-A, and DR-A, a single collection was suitable for genetic characterization of that apartment. As a result, a single kitchen sample from each subsequent apartment sampled was chosen as being representative of that population for all further analyses.

Within buildings. Pairwise G-tests were significant for all apartment pairs within the same building with the exception of complex CS, where adjoining apartments A and B were not significantly differentiated. Global $F_{\mathrm{ST}}$ values, calculated for each building independently, were all significant, with $95 \%$ confidence intervals not overlapping zero (range, 0.014-0.028) (Table 2). Individual pairwise $F_{\mathrm{ST}}$ values ranged from 0.009 to 0.037 , and with the exception of apartments CS-A and CS-B, all were significantly greater than zero. In all but one building, $\mathrm{CR}$, the lowest pairwise $F_{\mathrm{ST}}$ value was detected between the adjacent apartments. FCA analysis of samples collected in each building revealed comparable patterns of clearly separate clusters with little overlap (Fig. 3A-C).

Within complex. Significant departures from panmixia were detected at all three multi-building apartment complexes (FC, DR, and DRD) $(P<0.001$; $G$-test) when analyzed based on complex. Withincomplex global $F_{\mathrm{ST}}$ values were higher than withinbuilding values (Table 2); however, this increase was not significant $(P=0.077$; $t$-test, two sample assuming unequal variance). Comparable to within-building results, FCA within complex revealed distinct clusters with little overlap (Fig. 3E and F).

Across a city. Global $F_{\mathrm{ST}}$ across all Raleigh apartments sampled was significantly greater than zero (Table 2). Across sampled apartments, isolation by distance was detected. The $r^{2}$ value for the linear regression of genetic versus geographic distance was 0.160 (Fig. 4). A Mantel test was highly significant $(P<$ $0.001)$.

The NJ tree based on Chord distance revealed little genetic structure within Raleigh outside the scale of a single building (Fig. 5). Bootstrap support was generally weak throughout the tree. Similar tree topologies were observed when other distance measures (proportion of shared alleles and Nei's genetic distance) were used. The lack of association outside the level of a single building was mirrored by the results of STRUCTURE analysis. STRUCTURE analysis revealed that $B$. germanica from the apartments sampled comprise four genetic clusters $(K=4)$ based on calculation of $\Delta K$ (Evanno et al. 2005) (Fig. 6; Table 3). Only the samples from each of the single building complexes conformed to their own distinct clusters, with each building representing a genetic cluster. Analysis rerun excluding $B g-\mathrm{CO} 4$, due to the deviation

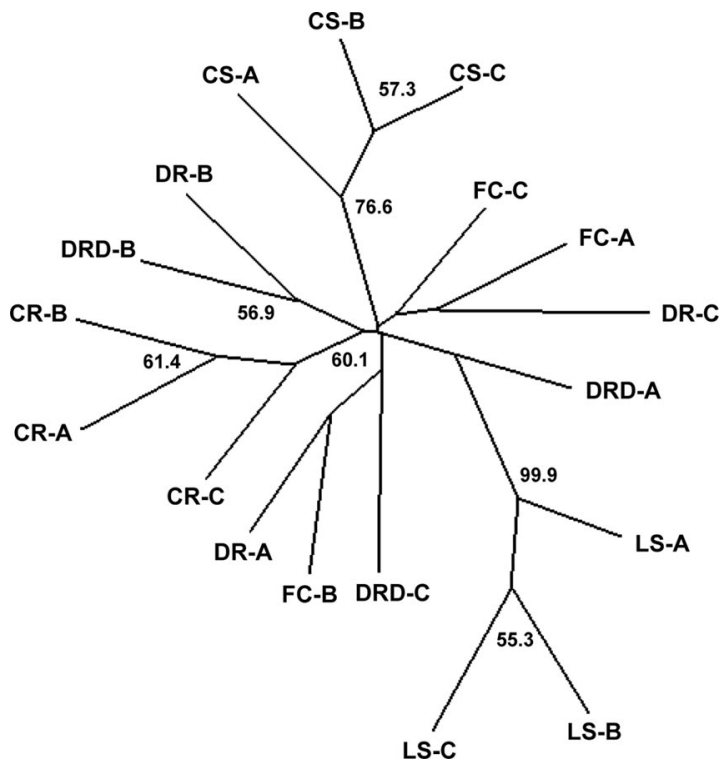

Fig. 5. Neighbor-joining tree based on chord distances for B. germanica apartment samples in Raleigh. Branch support is given as a percentage of 10,000 bootstraps. 


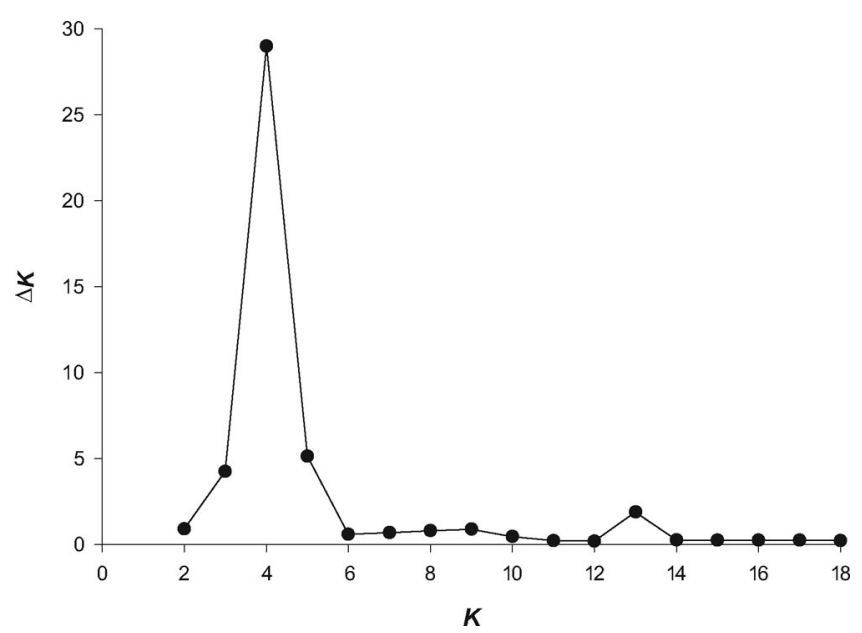

Fig. 6. Estimation of the most appropriate number of populations according to Evanno et al. (2005). $\Delta K$ estimates the second order rate of change of the log probability of data between successive values of $K$.

from HWE observed at this locus, revealed a similar pattern. When each cluster was reanalyzed separately, $K=1$ was observed.

Under the qualitative mode-shift test for detecting a population bottleneck, samples from four apartments were found to have a distorted (i.e., collapsed L) allelic frequency distribution, characterized by a poor representation of low frequency allelic classes (Table 2). Results of the Wilcoxon sign rank test, testing for a statistically significant excess of heterozygosity at microsatellite loci, revealed an excess in two samples with the shifted modes, and in three additional samples (Table 2).

\section{Discussion}

This study represents the most thorough investigation to date of the population genetic structure of $B$.

Table 3. Proportion of membership, according to the Bayesian clustering method STRUCTURE, for each predefined pop to each of the four inferred clusters identified by DK (Fig. 6)

\begin{tabular}{lccccr}
\hline \hline & \multicolumn{4}{c}{ Inferred cluster } & \multirow{2}{*}{$N$} \\
\cline { 2 - 5 } Sample ID & 1 & 2 & 3 & 4 & \\
\hline FC-A & 0.459 & 0.163 & 0.295 & 0.083 & 30 \\
FC-B & 0.470 & 0.281 & 0.212 & 0.037 & 30 \\
FC-C & $\mathbf{0 . 5 7 9}$ & 0.254 & 0.13 & 0.037 & 30 \\
DR-A & 0.469 & 0.334 & 0.172 & 0.025 & 30 \\
DR-B & 0.314 & 0.147 & 0.494 & 0.045 & 30 \\
DR-C & 0.383 & 0.294 & 0.265 & 0.058 & 30 \\
DRD-A & 0.398 & 0.293 & 0.269 & 0.040 & 30 \\
DRD-B & 0.269 & 0.134 & 0.488 & 0.109 & 30 \\
DRD-C & 0.367 & 0.380 & 0.214 & 0.039 & 30 \\
CR-A & 0.052 & 0.055 & 0.111 & $\mathbf{0 . 7 8 2}$ & 29 \\
CR-B & 0.047 & 0.031 & 0.039 & $\mathbf{0 . 8 8 3}$ & 30 \\
CR-C & 0.057 & 0.075 & 0.093 & $\mathbf{0 . 7 7 5}$ & 30 \\
CS-A & 0.139 & 0.123 & $\mathbf{0 . 7 0 8}$ & 0.030 & 30 \\
CS-B & 0.221 & 0.126 & $\mathbf{0 . 6 1 2}$ & 0.041 & 29 \\
CS-C & 0.135 & 0.136 & $\mathbf{0 . 6 9 0}$ & 0.039 & 30 \\
LS-A & 0.172 & $\mathbf{0 . 6 4 6}$ & 0.127 & 0.055 & 29 \\
LS-B & 0.131 & $\mathbf{0 . 6 7 0}$ & 0.111 & 0.088 & 30 \\
LS-C & 0.172 & $\mathbf{0 . 6 9 8}$ & 0.098 & 0.032 & 30 \\
\hline
\end{tabular}

Assignment scores $>50 \%$ indicated in bold. germanica at hierarchical levels spanning spatial scales from the apartment level to across a city. Previous studies have demonstrated the potential for dispersal within and between apartments (Owens and Bennett 1982; Akers and Robinson 1983; Runstrom and Bennett 1984, 1990); however, estimates of the distance and frequency of dispersal have varied greatly due to low recapture rates. In addition, using mark-recapture methods a direct association of such dispersal to gene flow is impossible. We used highly polymorphic microsatellite markers to assess the level of gene flow between apartments, a measure not possible with mark-recapture techniques (Slatkin 1987), offering greater resolution than markers used in previous population genetic studies of $B$. germanica (Cloarec et al. 1999, Jobet et al. 2000).

Overall genetic diversity across all sampled apartments was comparable to, and in some cases higher than, samples collected within swine farms in North Carolina (Booth et al. 2007). Given that B. germanica is highly sensitive to changes in environmental conditions, undergo genetic bottlenecks, possibly as a result of insecticide treatments and/or tenant turnover, and are sensitive to the availability of food and water (Schal and Hamilton 1990), such moderate to high levels of within-apartment diversity is surprising ( $>6$ alleles per locus and $H_{\mathrm{O}}=0.63$ ). How the diversity reported here might differ from a hypothetical native source population cannot be answered because no "natural," noncommensal populations of B. germanica have been found. Given the cosmopolitan distribution of this species, we may assume that the observed levels of genetic diversity are sufficient for both population establishment and growth.

Overall, only three apartment samples exhibited deviations from HWE. These deviations were not associated with particular loci and therefore are likely to have resulted from sampling error. This is supported by the fact that only a single sample of this group (FC-C) also exhibited the genetic signature of a recent genetic bottleneck. Frequent fluctuations in pop- 
ulation sizes are likely to contribute to departures from equilibrium; however, these would also be expected to have the same average effect on all loci (Hedrick 2005), a state not observed in any of our $B$. germanica samples. The fact that relatively few apartment samples were found to exhibit the genetic signature of a recent bottleneck, and alternatively a population expansion (through the detection of a deficit of heterozygotes), suggests that once established, population size remains sufficiently large to escape the effects of genetic drift.

Within Apartment Differentiation. No departures from panmixia were detected between cockroach samples collected within various rooms in single apartments, as determined through nonsignificant $G$-tests and pairwise/overall $F_{\mathrm{ST}}$ values. Interestingly, however, FCA plots do suggest that samples collected at different times (e.g., vacuum sample were collected during the day), or those from adjacent rooms, may show some degree of site fidelity. The highly gregarious nature of B. germanica (Amé et al. 2006), reported refuge loyalty (Denzer et al. 1988), and tendency to use the most closely available food source (Bret and Ross 1985, Silverman 1986, Rivault and Cloarec 1991, Durier and Rivault 2001) make it possible that cockroaches from various rooms may represent separate reproductive centers. That is to say, that aggregations within separate rooms of an apartment may originate from a common ancestral aggregation (most likely linked to the kitchen); however, after population expansion and subsequent dispersal, aggregations may form in different rooms within the apartment with limited gene exchange in relation to that occurring within aggregations. If true, it would suggest that mating is highly restricted to within aggregations, with mating between aggregations limited but sufficient to maintain apparent panmixia.

Within Building Differentiation. Significant departures from panmixia were observed between all but one pair of adjacent apartments and between all nonadjacent apartments within the same building, indicating that gene flow had either not occurred recently or had not involved sufficient numbers of individuals to counteract genetic differentiation. Although gene flow was not prevalent enough to lead to panmixia between these apartments, it is possible that infestations resulted from an initial founding event of a small but genetically diverse propagule, as supported by the detection of more than four alleles at most loci, followed by population expansion into adjacent apartments. Over time, due to aggregation behavior, refuge fidelity, the tendency to remain close to a reliable food source, and the avoidance of unfamiliar areas (Durier and Rivault 2003), B. germanica over increasing spatial scales within a building diverge genetically. This hypothesis is supported by the low but significant withinbuilding $F_{\mathrm{ST}} \mathrm{s}$ and the consistent clustering of withinbuilding samples in both the topology of the NJ tree and STRUCTURE analysis. Although it is possible that genetic divergence may increase periodically between apartments within a building after the introduction of cockroaches from other populations, an event that may then result in an increase in the genetic diversity within the building, we did not detect the genetic signature of either population admixture or expansion within any sample.

Differentiation Beyond Buildings. According to Roth (1985), the outdoor environment is considered unsuitable for B. germanica movement and population establishment. It may therefore effectively act as a physical barrier to active dispersal, limiting the potential for gene flow to events linked with humanmediated movement. Although such events may be rare, within-apartment-complex movements may be more common than between-complex movements, as suggested by the lower within-complex $F_{\mathrm{ST}} \mathrm{s}$ compared with the global $F_{\mathrm{ST}}$ considering all samples. This result is contradicted, however, by the failure to detect clustering based on apartment complex of origin within the topology of the NJ tree (Fig. 5). Furthermore, multibuilding samples collected within complexes did not associate within unique clusters following STRUCTURE analysis. In fact, cockroaches collected within all three complexes (FC, DR, and DRD) seemed to share genetic ancestry across each multibuilding complex (Table 3 ). This finding is somewhat perplexing as it suggests that human-mediated dispersal of B. germanica occurs no more frequently between buildings within an apartment complex than between distant apartment complexes. This then stands in contrast to the results of the Mantel test, which found that genetic distance between B. germanica populations was significantly correlated with geographic distance across the 18 Raleigh apartment samples. Removal of the within-building comparisons, which are nevertheless significantly differentiated, does not affect the result, with significance observed at the $<0.025$ level (data not shown).

Conclusions and Implications. Overall, the most reliable estimate of recent gene flow between our sampled apartments may be given by the pairwise G-tests, which identified 17 populations, with only adjacent apartments CS-A and CS-B not being differentiated from one another. In a simulation study, Waples and Gaggiotti (2006) found that the pairwise $G$-test was more powerful for estimating the true number of populations than assignment tests, particularly when gene flow was high or differentiation was low, or when a small number of markers or samples were used. That being said, within apartment buildings, pairwise $F_{\mathrm{ST}}$ estimates indicate that samples share a recent common ancestry with subsequent differentiation potentially resulting from the aggregation behavior exhibited by B. germanica.

These results have important implications to cockroach and allergen mitigation efforts within multiunit properties. The spatial coverage of most pest control treatments generally targets only the kitchen and bathroom, and only recent interventions with baits have treated the whole home. The latter have resulted in highly efficacious reductions in cockroaches and surprisingly large and unparalleled reductions in allergen concentrations throughout the home (Arbes et al. 2003, 2004; Sever et al. 2007; R.G.S. and C.S., un- 
published). Taking these findings into consideration, it is likely that within properties B. germanica aggregations from different rooms follow a metapopulation model (i.e., a population consisting of a network of partially isolated smaller populations). Thus, although they share a recent genetic ancestry, they exist essentially as independent units. Although treatment of the kitchen and bathroom may effectively eliminate cockroaches within these rooms, aggregations within adjacent untreated rooms may remain relatively unharmed, and therefore available to reseed other rooms over time with individuals of comparable genetic backgrounds to those lost. Furthermore, it is evident that despite being genetically differentiated, samples from different apartments are likely to be linked to a common ancestral source population within that building, albeit with infrequent additions over time. Therefore, given the active dispersal identified in earlier studies (Owens and Bennett 1982; Akers and Robinson 1983; Runstrom and Bennett 1984, 1990; Rivault 1990), it is likely that after the elimination of cockroaches from a room/apartment, reinfestation will result from the active dispersal of cockroaches from adjacent apartments. All infested apartments within a building therefore require simultaneous monitoring. The effort required for such building-wide control is likely to be substantial. If the goal is to reduce allergen levels and therefore improve public health, the aim must be to eliminate the cockroach populations, not simply to reduce them (Gore and Schal 2007).

\section{Acknowledgments}

We thank Paul Labadie for technical assistance. This project was supported in part by the National Research Initiative of the USDA Cooperative State Research, Education, and Extension Service (grant 2004-35302-14880); the USDA-Risk Avoidance and Mitigation Program (grant 200551101-02388); the Blanton J. Whitmire endowment at North Carolina State University; and a scholarship from the North Carolina Pest Management Association. D.V.M.'s research was supported in part by the Presidium of Russian Academy of Sciences grant "Biodiversity" (subprogram "Dynamics of Gene Pools"), and the Foundation for Basic Research (grants 08-04-01402-a and 09-04-01113-a).

\section{References Cited}

Akers, R. C., and W. H. Robinson. 1983. Comparison of movement behavior of 3 strains of German cockroach, Blattella germanica. Entomol. Exp. Appl. 34: 143-147.

Amé, J. M., J. Halloy, C. Rivault, C. Detrain, and J. L. Deneubourg. 2006. Collegial decision making based on social amplification leads to optimal group formation. Proc. Natl. Acad. Sci. U.S.A. 103: 5835-5840.

Appel, A. G. 1995. Blattella and related species. In M. K. Rust, J. M. Owens, and D. A. Reierson [eds.], Understanding and controlling the German cockroach. Oxford University Press, New York.

Arbes, S. J., M. Sever, J. Mehta, J. C. Gore, C. Schal, B. Vaughn, H. Mitchell, and D. C. Zeldin. 2004. Abatement of cockroach allergens (Bla g 1 and Bla g 2) in lowincome, urban housing: month 12 continuation results. J. Allergy Clin. Immunol. 113: 109-114.
Arbes, S. J., M. Sever, J. Archer, E. H. Long, J. C. Gore, C. Schal, M. Walter, B. Nuebler, B. Vaughn, H. Mitchell, et al. 2003. Abatement of cockroach allergen (Bla g 1) in low-income, urban housing: a randomized controlled trial. J. Allergy Clin. Immunol. 112: 339-345.

Belkhir, K., P. Borsa, L. Chikhi, N. Raufaste, and F. Bonhomme. 1996-2004. GENETIX 4.05, Windows TM software for population genetics, version 4.05, Laboratoire Génome, Populations, Interactions, CNRS UMR 5000, Université Montpellier II, Montpellier, France.

Booth, W., S. M. Bogdanowicz, P. A. Prodohl, R. G. Harrison, C. Schal, and E. L. Vargo. 2007. Identification and characterization of 10 polymorphic microsatellite loci in the German cockroach, Blattella germanica. Mol. Ecol. Notes 7: $648-650$.

Bret, B. L., and M. H. Ross. 1985. A laboratory study of German cockroach dispersal (Dictyoptera, Blattellidae). Proc. Entomol. Soc. Wash. 87: 448-455.

Cavalli-Sforza, L. L., and A.W.F. Edwards. 1967. Phylogenetic analysis: models and estimation procedures. Evolution 21: 550-570.

Cloarec, A., C. Rivault, and M. L. Cariou. 1999. Genetic population structure of the German cockroach, Blattella germanica: absence of geographical variation. Entomol. Exp. Appl. 92: 311-319.

Cloarec, A., C. Rivault, F. Fontaine, and A. Leguyader. 1992. Cockroaches as carriers of bacteria in multifamily dwellings. Epidemiol. Infect. 109: 483-490.

Cornuet, J. M., and G. Luikart. 1996. Description and power analysis of two tests for detecting recent population bottlenecks from allele frequency data. Genetics 144: 20012014.

Denzer, D. J., M.E.A. Fuchs, and G. Stein. 1988. Behavioral studies on Blattella germanica L.: radius of action and loyalty to the refuge. J. Appl. Entomol. 105: 330-343.

Di Rienzo, A., A. C. Peterson, J. C. Garza, A. M. Valdes, M. Slatkin, and N. B. Freimer. 1994. Mutational processes of simple sequence repeat loci in human populations. Proc. Natl. Acad. Sci. U.S.A. 91: 3166-3170.

Durier, V., and C. Rivault. 2001. Effects of spatial knowledge and feeding experience on foraging choices in German cockroaches. Anim. Behav. 62: 681-688.

Durier, V., and C. Rivault. 2003. Exploitation of home range and spatial distribution of resources in German cockroaches (Dictyoptera: Blattellidae). J. Econ. Entomol. 96: 1832-1837.

Eggleston, P. A., D. Rosenstreich, M. Lynn, P. Gergen, D. Baker, M. Kattan, K. M. Mortimer, H. Mitchell, D. Ownby, R. Slavin, and F. Malveaux. 1998. Relationship of indoor allergen exposure to skin test sensitivity in inner-city children with asthma. J. Allergy Clin. Immunol. 102: 563-570.

Elgderi, R. M., K. S. Ghenghesh, and N. Berbash. 2006. Carriage by the German cockroach (Blattella germanica) of multiple-antibiotic-resistant bacteria that are potentially pathogenic to humans, in hospitals and households in Tripoli, Libya. Ann. Trop. Med. Parasitol. 100: 55-62.

Evanno, G., S. Regnaut, and J. Goudet. 2005. Detecting the number of clusters of individuals using the software STRUCTURE: a simulation study. Mol. Ecol. 14: 26112620 .

Felsenstein, J. 1985a. Confidence limits on phylogenies: an approach using the bootstrap. Evolution 39: 783-791.

Felsenstein, J. 1985b. PHYLIP: phylogenetic inference programs, version 3.572. Department of Genetics, University of Washington, Seattle, WA. 
Fotedar, R., and U. Banerjee. 1992. Nosocomial fungal-infections: Study of the possible role of cockroaches (Blattella germanica) as vectors. Acta Trop. 50: 339-343.

Fotedar, R., U. B. Shriniwas, and A. Verma. 1991. Cockroaches (Blattella germanica) as carriers of microorganisms of medical importance in hospitals. Epidemiol. Infect. 107: 181-187.

Gore, J. C., and C. Schal. 2007. Cockroach allergen biology and mitigation in the indoor environment. Annu. Rev. Entomol. 52: 439-463.

Goudet, J. 1995. FSTAT (Version 1.2): a computer program to calculate F-statistics. J. Hered. 86: 485-486.

Goudet, J., M. Raymond, T. deMeeus, and F. Rousset. 1996. Testing differentiation in diploid populations. Genetics 144: 1933-1940.

Graczyk, T. K., R. Knight, and L. Tamang. 2005. Mechanical transmission of human protozoan parasites by insects. Clin. Microbiol. Rev. 18: 128-132.

Hedrick, P. W. 2005. Genetics of populations. Jones and Bartlett Publishers, Boston, MA.

Jakobsson, M., and N. A. Rosenberg. 2007. CLUMPP: a cluster matching and permutation program for dealing with label switching and multimodality in analysis of population structure. Bioinformatics 23: 1801-1806.

Jobet, E., P. Durand, J. Langand, C.D.M. Muller-Graf, J. P. Hugot, M. E. Bougnoux, C. Rivault, A. Cloarec, and S. Morand. 2000. Comparative genetic diversity of parasites and their hosts: population structure of an urban cockroach and its haplo-diploid parasite (oxyuroid nematode). Mol. Ecol. 9: 481-486.

Lewis, P. O., and D. Zaykin. 2001. Genetic data analysis: computer program for the analysis of allelic data, version $1.0(\mathrm{~d} 16 \mathrm{c})$. Free program distributed by the authors over the internet. (http://lewis.eeb.uconn.edu/lewishome/ software.html).

Liedloff, A. 1999. MANTEL, Mantel nonparametric test calculator, version 2. (http://www.sci.qut.edu.au/nrs/ mantel.htm).

Luikart, G., F. W. Allendorf, J. M. Cornuet, and W. B. Sherwin. 1998. Distortion of allele frequency distributions provides a test for recent population bottlenecks. J. Hered. 89: 238-247.

Mantel, N. 1967. Detection of disease clustering and a generalized regression approach. Cancer Res. 27: 209-214.

Minch, E., A. Ruiz-Linares, D. Goldstein, M. Feldman, and L. L. Cavalli-Sforza. 1995. Microsat: a computer program for calculating various statistics on microsatellite allele data, version 1.4d. (http://hpgl.stanford.edu/ projects/microsat/ ).

Owens, J. M., and G. W. Bennett. 1982. German cockroach (Orthoptera, Blattellidae) movement within and between urban apartments. J. Econ. Entomol. 75: 570-573.

Pai, H. H., Y. C. Ko, and E. R. Chen. 2003. Cockroaches (Periplaneta americana and Blattella germanica) as potential mechanical disseminators of Entamoeba histolytica. Acta Trop. 87: 355-359.

Pai, H. H., W. C. Chen, and C. F. Peng. 2005. Isolation of bacteria with antibiotic resistance from household cockroaches (Periplaneta americana and Blattella germanica). Acta Trop. 93: 259-265.

Pritchard, J. K., M. Stephens, and P. Donnelly. 2000. Inference of population structure using multilocus genotype data. Genetics 155: 945-959.
Raymond, M., and F. Rousset. 1995. Genepop (version 1.2): population genetics software for exact tests and ecumenicism. J. Hered. 86: 248-249.

Rivault, C. 1990. Distribution dynamics of Blattella germanica in a closed urban environment. Entomol. Exp. Appl. 57: 85-91.

Rivault, C., and A. Cloarec. 1991. Exploitation of food resources by the cockroach Blattella germanica in an urban habitat. Entomol. Exp. Appl. 61: 149-158.

Rosenstreich, D., P. Eggleston, M. Kattan, D. Baker, R. Slavin, P. Gergen, H. Mitchell, K. McNiff-Mortimer, H. Lynn, D. Ownloy, and F. Malveaux. 1997. The role of cockroach allergy and exposure to cockroach allergen in causing morbidity among inner-city children with asthma. N. Engl. J. Med. 336: 1356-1363.

Roth, L. M. 1985. Taxonomic revision of the genus Blattella Caudell (Dictyoptera, Blattaria: Blattellidae). Entomol. Scand. Suppl. 22: 1-221.

Rousset, F. 2008. GENEPOP' 007: a complete re-implementation of the GENEPOP software for Windows and Linux. Mol. Ecol. Resour. 8: 103-106.

Runstrom, E. S., and G. W. Bennett. 1984. Movement of German cockroaches (Orthoptera, Blattellidae) as influenced by structural features of low-income apartments. J. Econ. Entomol. 77: 407-411.

Runstrom, E. S., and G. W. Bennett. 1990. Distribution and movement patterns of German cockroaches (Dictyoptera, Blattellidae) within apartment buildings. J. Med. Entomol. 27: 515-518.

Schal, C., and R. L. Hamilton. 1990. Integrated suppression of synanthropic cockroaches. Annu. Rev. Entomol. 35: $521-551$

Sever, M. L., S. J. Arbes, J. C. Gore, R. G. Santangelo, B. Vaughn, H. Mitchell, C. Schal, and D. C. Zeldin. 2007. Cockroach allergen reduction by cockroach control alone in low-income urban homes: a randomized control trial. J. Allergy Clin. Immunol. 120: 849-855.

Silverman, J. 1986. Adult German cockroach (Orthoptera, Blattellidae) feeding and drinking behavior as a function of density and harborage-to-resource distance. Environ. Entomol. 15: 198-204.

Slatkin, M. 1987. Gene flow and the geographic structure of natural populations. Science 236: 787-792.

Takezaki, N., and M. Nei. 1996. Genetic distances and reconstruction of phylogenetic trees from microsatellite DNA. Genetics 144: 389-399.

Van Oosterhout, C., W. F. Hutchinson, D.P.M. Wills, and P. Shipley. 2004. MICRO-CHECKER: software for identifying and correcting genotyping errors in microsatellite data. Mol. Ecol. Notes 4: 535-538.

Waples, R. S., and O. Gaggiotti. 2006. What is a population? An empirical evaluation of some genetic methods for identifying the number of gene pools and their degree of connectivity. Mol. Ecol. 15: 1419-1439.

Weir, B. S., and C. C. Cockerham. 1984. Estimating F-statistics for the analysis of population structure. Evolution 38: $1358-1370$

Zurek, L., and C. Schal. 2004. Evaluation of the German cockroach (Blattella germanica) as a vector for verotoxigenic Escherichia coli F18 in confined swine production. Vet. Microbiol. 101: 263-267.

Received 10 February 2009; accepted 22 February 2010. 